\title{
细胞核定位的萗酰亚胺类衍生物的合成及抗肿瘤作用研究
}

\author{
戎瑞雪 $a, b$ 李基民 $a$ 李耀文 ${ }^{a}$ 郭啸宇 $a$ 王 冲 $b$ 李艳军 $c$ \\ 李金梅 ${ }^{d}$ 韩宝君 $c$ 曹志然 $a$ 王克让 $* b$ 李小六*,b

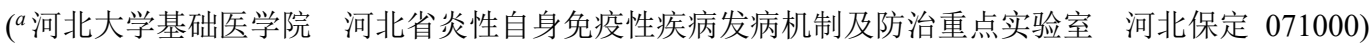 \\ ( $b$ 河北大学化学与环境科学学院 药物化学与分子诊断教育部重点实验室 \\ 河北省化学生物学重点实验室 河北保定 071002) \\ (c 保定市第一中心医院骨四科 河北保定 071000) \\ ( $d$ 保定市第一中心医院病理科 河北保定 071000)
}

\begin{abstract}
摘要 以 4-溴-1,8-萗酐为原料, 经亲核取代反应合成了系列手性氨基醇修饰的萘酰亚胺衍生物 NI1 NI8. 四甲基偶氮 唑蓝(MTT)法研究了其细胞毒性, 发现含有伯羟基氨基醇修饰的菜酰亚胺衍生物中 $R$ 型的细胞毒性好于 $S$ 型异构体. 经 紫外光谱、荧光光谱和激光共聚焦实验研究了化合物( $R$ )-2-(2-(二甲基氨基)乙基)-6-((1-羟基-2-丙烷基)氨基)-荎酰亚胺 (NI1)和(S)-2-(2-(二甲基氨基)乙基)-6-((1-羟基-2-丙烷基)氨基)-菜酰亚胺(NI2)与 DNA 分子和 HeLa 细胞的相互作用，发

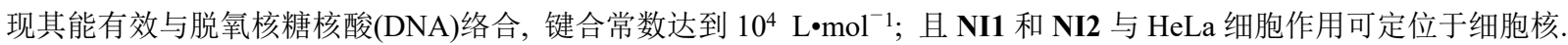
流式细胞实验结果显示 NI1 和 NI2 能够使细胞周期阻滞于 S 期而抑制细胞增殖. 小鼠体内血液毒性结果显示 NI1 对血. 液中的红细胞、白细胞和血小板无明显影响.
\end{abstract}

关键词 荎酰亚胺; 细胞核; 手性; 细胞周期

\section{Synthesis and Anti-tumor Effects of Naphthalimide Derivatives Targeted in Cell Nucleus}

\author{
Rong, Ruixue ${ }^{a, b} \quad$ Li, Jimin $^{a} \quad$ Li, Yaowen $^{a} \quad$ Guo, Xiaoyu ${ }^{a} \quad$ Wang, Chong $^{b} \quad$ Li, Yanjun $^{c}$ \\ Li, Jinmei $^{d} \quad$ Han, Baojun $^{c} \quad$ Cao, Zhiran $^{a} \quad$ Wang, Kerang*,b Li, Xiaoliu*,b \\ $\left({ }^{a}\right.$ College of Basic Medicine, Key Laboratory of Pathogenesis Mechanism and Control of Inflammatory-autoimmune Diseases \\ in Hebei Province, Hebei University, Baoding, Hebei 071000) \\ ( ${ }^{b}$ College of Chemistry and Environmental Science, Key Laboratory of Medicinal Chemistry and Molecular Diagnosis (Min- \\ istry of Education), Key Laboratory of Chemical Biology of Hebei Province, Hebei University, Baoding, Hebei 071002) \\ ( ${ }^{c}$ Forth Department of Orthopedic, Baoding First Central Hospital, Baoding, Hebei 071000) \\ ( ${ }^{d}$ Department of Pathology, Baoding First Central Hospital, Baoding, Hebei 071000)
}

\begin{abstract}
A series of novel chiral amino alcohol modified naphthalimide derivatives NI1 $\sim$ NI8 were designed and synthesized by nucleophilic substitution reaction. Furthermore, their cytotoxicities were studied by thiazolyl blue tetrazolium bromide (MTT) method. The cytotoxicity of the $R$-type isomer in the naphthalimide derivatives with primary hydroxyl amino alcohol modification was better than that of the $S$-type isomer. In addition, deoxyribonucleic acid (DNA) binding interactions and fluorescence imaging of $(R)-2-(2-($ dimethylamino)ethyl)-6-((1-hydroxypropan-2-yl)amino)-naphthalimide (NI1) and (S)-2-(2-(dimethylamino)ethyl)-6-((1-hydroxypropan-2-yl)amino)-naphthalimid (NI2) with Ct-DNA and HeLa cells were investigated. NI1 and NI2 showed strong binding interactions with Ct-DNA with a bonding constant of $10^{4} \mathrm{~L} / \mathrm{mol}$. And NI1 and NI2 exhibited nucleus-targeting imaging for HeLa cells. NI1 and NI2 mainly arrested the S phase. Moreover, the hematoxic
\end{abstract}

\footnotetext{
* Corresponding authors. E-mail: kerangwang@hbu.edu.cn; lixl@hbu.edu.cn

Received August 11, 2020; revised September 15, 2020; published online December 31, 2020.

Project supported by the National Natural Science Foundation of China (Nos. 21572044, 21778013), the Natural Science Foundation of Hebei Province (No. B2017201193), the Foundation of Health Commission of Hebei Province (No. 20202217), the Innovation Project of Students (Nos. 201910075009, 2020338) and Hebei University High-level Talent Research Startup Project (No. 521000981311).

国家自然科学基金(Nos. 21572044, 21778013)、河北省自然科学基金(No. B2017201193)、河北省卫生健康委员会基金(No. 20202217)、大学生创新项 目(Nos. 201910075009, 2020338)及河北大学高层次人才科研启动基金(No. 521000981311)资助项目.
} 
results of NI1 in mice showed no significant effects on the red blood cells, white blood cells and platelets in the blood.

Keywords naphthalimide; nucleus; chiral; cell cycle

细胞核作为高级生物体的高度专业化的细胞器 ${ }^{[1]}$, 具有储存遗传物质和协调细胞活动的作用，与转录 ${ }^{[2]}$ 、 细胞分裂 ${ }^{[3]}$ 及基因表达等密切相关 ${ }^{[4]}$. 与正常细胞相比, 肿瘤细胞增殖快，代谢活跃，其细胞核的形态结构有很 多的异常，如细胞核通常较大，核质比增高 ${ }^{[5]}$; 细胞核 呈现形状拉长、边缘呈锯齿状、凹陷、长芽、分叶及弯 月形等畸形; 核膜增厚且呈不规则状，可出现小泡、小 囊状突起等现象; 核孔的数目增加; 核仁大且数目较 多 ${ }^{[6]}$. 如在骨髓瘤细胞中, 甚至出现细胞核分裂但细胞 质不分裂而形成的双核细胞(四倍体). 众所周知, 脱氧 核糖核酸(DNA)的含量与肿瘤细胞的增殖密切相关 ${ }^{[7]}$, 而细胞核是 DNA 的主要载体. 因此, 以 DNA 为靶点 ${ }^{[8]}$, 研究细胞核的形态与抗肿瘤作用的关系在肿瘤治疗方 面具有重要意义.

近年来, 利用苂光染料与 DNA 分子的作用实时监 测细胞核的形态和功能的研究取得了一定进展 ${ }^{[9]}$. Zhang 和 Guo 等 ${ }^{[10]}$ 合成了基于䒬酰亚胺类的谷氨酰转移 酶(GGT)的双光子荧光探针, 通过单/双光子显微镜和手 持紫外灯实现了对癌细胞的 DNA 损伤的实时成像. 该 研究结果为肿瘤形成和进展过程的研究提供了重要的 参考价值. 然而, 基于细胞核形态与抗肿瘤作用关系的 研究还较少. 要系统研究细胞核形态与抗肿瘤作用的关 系, 苂光染料分子具有关键性的作用. 该荧光染料分子 既具有好的苂光特征, 又要具有好的抗肿瘤作用. 荎酰 亚胺类分子是研究细胞核形态与抗肿瘤作用的理想选 择，其作为 DNA 嵌入剂，不仅具有良好的荧光特征 ${ }^{[11]}$, 而且具有好的抗肿瘤作用 ${ }^{[12]}$. 䒬酰亚胺类衍生物具有 较强的荧光特性 ${ }^{[13]}$, 被广泛用于亚细胞器的荧光成像,

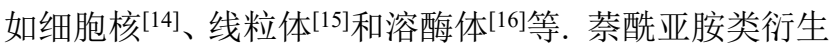
物作为抗肿瘤活性的研究取得了突出成果, 系列化合物 (如氨荎非特、米托菜胺、依利奈法德、双荎法德和 UNBS5162)进入临床研究 ${ }^{[17]}$. 近年来, 我们课题组在基 于荥酰亚胺类衍生物的成像和肿瘤治疗方面做了初步 研究, 发现了溶酶体靶向的抗肿瘤分子[18]、细胞核和核 仁定位的抗肿瘤分子 [19]以及既具有成像作用又具有抗 肿瘤作用的化合物 ${ }^{[20]}$. 上述荎酰亚胺类衍生物都是 4-位 环状氨基醇或环状胺类修饰, 并且未发现完全定位于细 胞核的荎酰亚胺类衍生物.

因此，基于菜酰亚胺类衍生物良好的荧光特征和抗 肿瘤作用, 我们设计合成了系列手性氨基醇修饰的荎酰 亚胺衍生物 NI1 NI8 (Scheme 1), 手性氨基醇分子包 括 $(R)$-2-氨基-1-丙醇、 $(S)$-2-氨基-1-丙醇、 $(R)$-1-氨基-2丙醇、 $(S)$-1-氨基-2-丙醇、 $(R)$-2-氨基丁醇、 $(S)$-2-氨基丁
醇、 $(R)$-3-氨基-1,2-丙二醇和 $(S)$-3-氨基-1,2-丙二醇等, 通过四甲基偶氮唑蓝(MTT)法研究了 NI1 NI8 的抗肿 瘤活性. 我们系统地研究了(R)-2-(2-(二甲基氨基)乙 基 )-6-((1- 羟基-2-丙烷基) 氨基)-䒬酰亚胺 (NI1) 和 (S)-2-(2-(二甲基氨基)乙基)-6-((1-羟基-2-丙烷基)氨基)䒺酰亚胺(NI2)与 DNA 分子的相互作用、苂光成像和细 胞周期. 研究结果发现 NI1 和 NI2 显示了好的细胞毒性, 可选择性定位于细胞核, 并将细胞有效阻滞于 $\mathrm{S}$ 期.
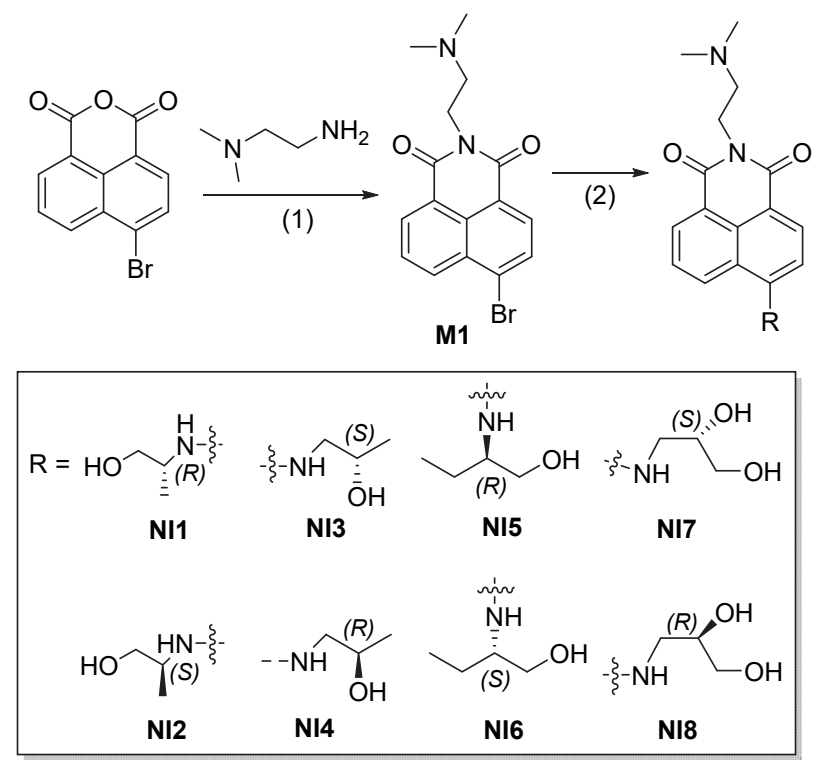

Reagents and conditions: (1) EtOH, reflux; (2) (R)-2-amino-1-propanol, (S)-2-amino-1-propanol, (R)-1-amino-2-propanol, (S)-1amino-2-propanol, $(R)$-2-aminobutanol, $(S)$-2-aminobutanol, $(R)$-3amino-1,2-propanediol or (S)-3-amino-1,2-propanediol, $\mathrm{HOCH}_{2}$ $\mathrm{CH}_{2} \mathrm{OCH}_{3}$, DIPEA, reflux

图式 1 NI1 NI8 的合成路线

Scheme 1 Synthetic route of compounds NI1 NI8

\section{1 结果与讨论}

\section{1 系列萗酰亚胺类衍生物的合成}

以 4-溴-1,8-荎酩为原料, 在乙醇溶液中与 $N, N$-二甲 基乙二胺反应得菜酰亚胺中间体 6-溴-2-（2-（二甲基氨 基）乙基）-2-甲基-䒬酰亚胺(M1) ${ }^{[21]}$ 进而与系列手性氨 基醇 $[(R)$-2-氨基-1-丙醇、 $(S)$-2-氨基-1-丙醇、 $(R)$-1-氨 基-2-丙醇、 $(S)$-1-氨基-2-丙醇、 $(R)$-2-氨基丁醇、 $(S)$-2氨基丁醇、 $(R)$-3-氨基-1,2-丙二醇和 $(S)$-3-氨基-1,2-丙二 醇]反应，制备了系列 4 位手性氨基醇修饰的芸酰亚胺 类衍生物 NI1 NI8 (Scheme 1). NI1 NI8 的结构经 NMR 和 HRMS 等表征. 


\section{2 细胞毒性研究}

通过四甲基偶氮唑盐微量酶反应比色法(简称 MTT 法), 研究了化合物 NI1 NI8 对人宫颈癌细胞 (HeLa)、 人乳腺癌细胞(MCF-7)、人肺癌细胞(A549)和人胃癌细 胞(MGC-803)的细胞毒性. 如表 1 所示, NI1 NI8 对 HeLa 和 A549 细胞的细胞毒性低于化合物对 MCF-7 和 MGC-803 的细胞毒性. 其中化合物 NI1、NI2、 ( $R$ )-2-(2-(二甲基氨基) 乙基)-6-((1-差基-2-丙烷基)氨 基)- $1 H$-苯并异喹啉-1,3(2H)-二酮(NI5)、(S)-2-(2-(二甲基 氨基)乙基)-6-((1-着基-2-丙烷基)氨基)- $1 H$-苯并异喹啉1,3(2H)-二酮(NI6)、(S)-6-((2,3-二羟丙基)氨基)-2-(2-(二 甲基氨基)乙基)- $1 H$-苯并异喹啉-1,3(2H)-二酮(NI7)和 ( $R$ )-6-((2,3-二羟丙基)氨基)-2-(2-(二甲基氨基)乙基)- $1 H$ 苯并异喹啉-1,3(2H)-二酮(NI8)修饰的手性氨基醇中都 含有伯羟基, $R$ 型的异构体的细胞毒性低于 $S$ 型的异构 体的细胞毒性. 而(S)-2-(2-(二甲基氨基)乙基)-6-((2-差 丙基) 氨基)- $1 H$ - 苯并异喹啉 $-1,3(2 H)$-二酮 (NI3) 和 ( $R$ )-2-(2-(二甲基氨基)乙基)-6-((2-差丙基)氨基)-1 $H$-苯 异喹啉-1,3(2H)-二酮(NI4)含有仲羟基的氨基醇分子中 $S$
型异构体的活性低于 $R$ 型异构体的活性. 其中, NI1 和 NI2 异构体对 HeLa 和 A549 细胞都具有强的细胞毒性, $\mathrm{IC}_{50}$ 值分别为 4.76、1.51、8.03 和 $3.47 \mu \mathrm{mol} / \mathrm{L}$, 活性好 于或接近阳性对照分子安奈菲特和顺铂.

\section{3 蒜酰亚胺衍生物与 DNA 作用研究}

鉴于化合物 NI1 和 NI2 对测试的四个肿瘤细胞都有 较强的细胞毒性, 尤其是对 HeLa 和 A549 细胞. 因此, 我们进一步研究了化合物 NI1 和 NI2 与小牛胸腺 DNA (Ct-DNA)的络合作用. 如图 1 所示, 随着 Ct-DNA 的加 入, 化合物 NI1 和 NI2 的最大吸收峰都从 $448.5 \mathrm{~nm}$ 红移 到 $458.0 \mathrm{~nm}$, 红移近 $10 \mathrm{~nm}$, 并伴随着吸收强度降低. 研 究结果说明化合物 NI1 和 NI2 的萗酰亚胺分子平面嵌入 到 DNA 的碱基中, 其作为 DNA 嵌入剂[22]与 Ct-DNA 有 效络合. 接着通过非线性拟合方法 ${ }^{[23]}$ 研究了化合物 NI1 和 NI2 与 Ct-DNA 作用的键合常数, 如图 1 插图所示, 其 键合常数分别是 $(1.25 \pm 0.50) \times 10^{4}$ 和 $(2.44 \pm 0.70) \times 10^{4}$ $\mathrm{L} \cdot \mathrm{mol}^{-1}$, 说明化合物 NI1 和 NI2 与 Ct-DNA 作用的键合 能力相当.

表 1 化合物 NI1 NI8 的细胞毒性

Table 1 Cytotoxic activity of compounds NI1 NI8

\begin{tabular}{ccccc}
\hline \multirow{2}{*}{ Compd. } & \multicolumn{3}{c}{$\mathrm{IC}_{50} /\left(\mu \mathrm{mol} \cdot \mathrm{L}^{-1}\right)$} \\
\cline { 2 - 5 } & HeLa & MCF-7 & A549 & MGC-803 \\
\hline NI1 $(R)$ & $\mathbf{4 . 7 6} \pm \mathbf{0 . 0 5}$ & $14.44 \pm 0.04$ & $\mathbf{1 . 5 1} \pm \mathbf{0 . 0 1}$ & $16.79 \pm 0.53$ \\
NI2 $(S)$ & $\mathbf{8 . 0 3} \pm \mathbf{0 . 0 3}$ & $32.59 \pm 0.01$ & $\mathbf{3 . 4 7} \pm \mathbf{0 . 0 6}$ & $28.38 \pm 0.49$ \\
NI3 $(S)$ & $3.99 \pm 0.05$ & $11.64 \pm 0.01$ & $4.33 \pm 0.04$ & $17.33 \pm 0.41$ \\
NI4 $(R)$ & $7.79 \pm 0.02$ & $17.69 \pm 0.04$ & $22.15 \pm 0.05$ & $29.14 \pm 0.33$ \\
NI5 $(R)$ & $6.42 \pm 0.02$ & $11.56 \pm 0.02$ & $5.86 \pm 0.04$ & $7.82 \pm 0.37$ \\
NI6 $(S)$ & $13.58 \pm 0.04$ & $25.40 \pm 0.01$ & $30.99 \pm 0.03$ & $19.04 \pm 0.34$ \\
NI7 $(S)$ & $11.89 \pm 0.04$ & $70.43 \pm 0.02$ & $17.66 \pm 0.01$ & $63.44 \pm 0.33$ \\
NI8 $(R)$ & $5.84 \pm 0.04$ & $71.05 \pm 0.02$ & $8.745 \pm 0.04$ & $34.71 \pm 0.35$ \\
Amonafide & $3.40 \pm 0.03$ & $6.79 \pm 0.02$ & $1.99 \pm 0.08$ & $3.23 \pm 0.43$ \\
Cisplatin & $6.45 \pm 0.03$ & $13.11 \pm 0.01$ & $17.00 \pm 0.01$ & $8.87 \pm 0.24$ \\
\hline
\end{tabular}
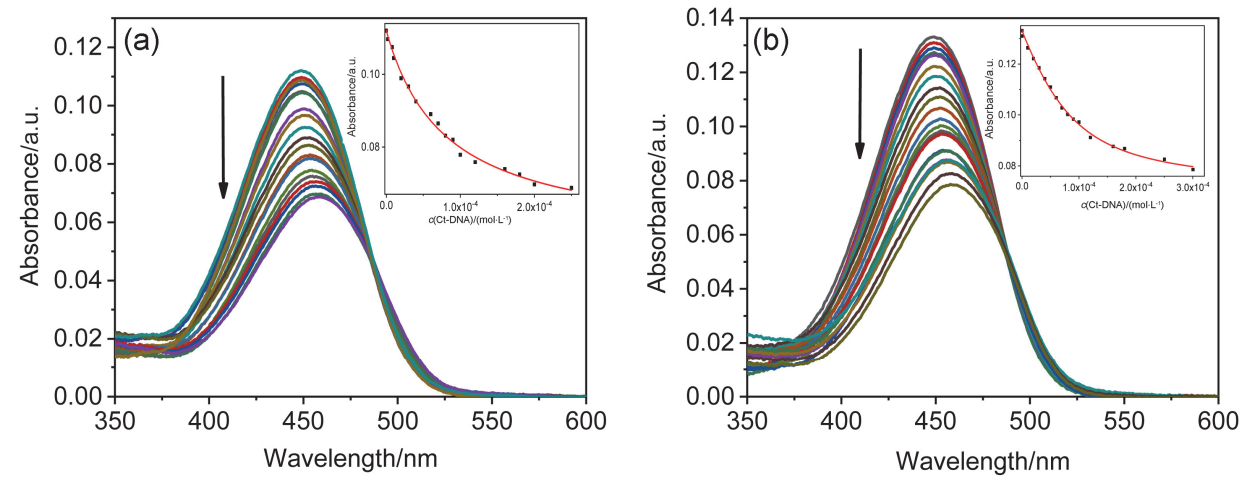

图 1 磷酸盐缓冲液中 $(10 \mathrm{mmol} / \mathrm{L}, \mathrm{pH} 7.4,50 \mathrm{mmol} / \mathrm{L} \mathrm{NaCl}) \mathbf{N I 1}\left(\mathrm{a}, 1 \times 10^{-5} \mathrm{~mol} / \mathrm{L}\right)$ 和 $\mathbf{N I 2}\left(\mathrm{b}, 1 \times 10^{-5} \mathrm{~mol} / \mathrm{L}\right)$ 与 $\mathrm{Ct}-\mathrm{DNA}$ 的作用的紫 外光谱

Figure 1 UV-Vis spectra of NI1 (a, $\left.1 \times 10^{-5} \mathrm{~mol} / \mathrm{L}\right)$ and NI2 (b, $\left.1 \times 10^{-5} \mathrm{~mol} / \mathrm{L}\right)$ binding with Ct-DNA in PBS buffer $(10 \mathrm{mmol} / \mathrm{L}, \mathrm{pH}$ $7.4,50 \mathrm{mmol} / \mathrm{L} \mathrm{NaCl})$

Inset: the fitting curve of NI1 and NI2 binding with Ct-DNA 
通过荣光光谱研究了化合物 NI1 和 NI2 与 Ct-DNA 的络合作用. 随着 Ct-DNA 的加入, 化合物 NI1 和 NI2 的苂光呈先升高再降低的趋势. 当 Ct-DNA 的物质的量 与 NI1 和 NI2 接近 1 时, 其苂光强度达到最大, 继续加 入 Ct-DNA 时, 其荧光强度逐渐降低. 这可能是由于加 入不同物质的量浓度的 DNA 分子时, 化合物 NI1 和 NI2 与其作用模式改变所致 ${ }^{[21]}$.

\section{4 慕酰亚胺衍生物与 HeLa 细胞荧光成像研究}

鉴于化合物 NI1 和 NI2 与 DNA 分子具有强的络合 作用, 因此, 我们推测其可能与肿瘤细胞的细胞核具有 定位作用. 如图 2 所示, 化合物 NI1 和 NI2 与 HeLa 细 胞孵育 $2 \mathrm{~h}$ 时, 可完全进入细胞核, 细胞核边缘光滑清 晰. 研究结果说明化合物 NI1 和 NI2 可定位于肿瘤细胞 的细胞核. 手性氨基醇的修饰, 既增加了化合物 NI1 和 NI2 的亲水性又增加了其正电性 ${ }^{[21]}$, 容易通过静电作用 增强细胞膜通透性, 有利于化合物 NI1 和 NI2 进入细胞 核. 接着, 研究了含有仲羟基氨基醇修饰的萗酰亚胺化
合物 NI3 与 HeLa 细胞的苂光成像, 化合物 NI3 可定位 于细胞核, 与 NI1 和 NI2 的实验结果一致. 研究结果说 明氨基醇的引入, 有利于菜酰亚胺衍生物进入细胞核, 增强化合物的细胞毒性.

\section{5 萗酰亚胺衍生物与 HeLa 细胞的周期研究}

鉴于化合物 NI1 和 NI2 与 DNA 分子具有较强的络 合作用，可在肿瘤细胞细胞核成像，我们推测其可影响 肿瘤细胞周期的改变. 其中, 细胞周期分为 $\mathrm{G} 1$ 期、 $\mathrm{S}$ 期、 $\mathrm{G} 2$ 期和 $\mathrm{M}$ 期, 而 $\mathrm{S}$ 期是 DNA 复制期[24], DNA 在这一 阶段合成完毕. 因此, 我们推测化合物 NI1 和 NI2 与 HeLa 作用可能将细胞周期阻滞在 $\mathrm{S}$ 期. 如图 3 所示, HeLa 细胞在加入化合物 NI1 和 NI2 之前的细胞周期分 别为 $\mathrm{G} 1 / \mathrm{G} 2=61.84 \% 、 \mathrm{G} 2 / \mathrm{M}=6.90 \%$ 和 $\mathrm{S}=31.27 \%$. 当 加入 $7.5 \mu \mathrm{mol} / \mathrm{L}$ 化合物 NI1 和 NI2 后, HeLa 细胞的细胞 周期发生明显变化，其中 $\mathrm{G} 1 / \mathrm{G} 2$ 期从 $61.84 \%$ 分别降低 到 $42.77 \%$ 和 $42.72 \%, \mathrm{G} 2 / \mathrm{M}$ 期从 $6.90 \%$ 升高到 $8.82 \%$ 和 $16.68 \%, \mathrm{~S}$ 期从 $31.27 \%$ 升高到 $48.42 \%$ 和 $40.60 \% \mathrm{~S}$ 期的

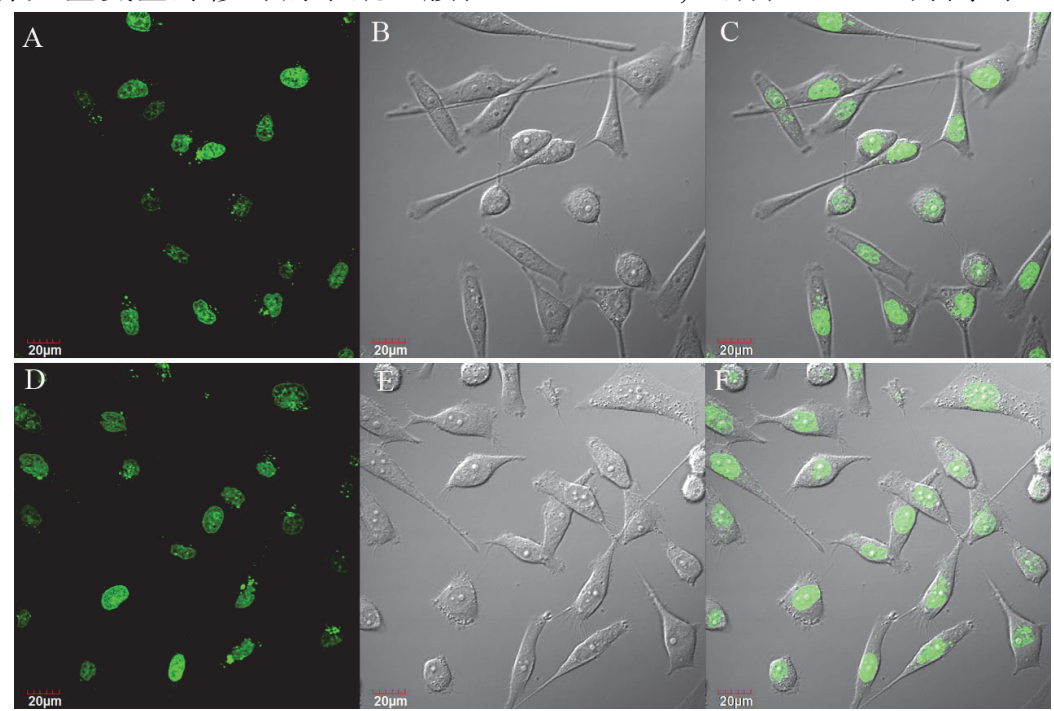

图 $2 \mathrm{NI1}\left(2 \mu \mathrm{mol} / \mathrm{L}, \lambda_{\mathrm{ex}}=405 \mathrm{~nm}\right)$ 和 NI2 $\left(2 \mu \mathrm{mol} / \mathrm{L}, \lambda_{\mathrm{ex}}=405 \mathrm{~nm}\right)$ 与 HeLa 细胞作用的苂光成像 $\left(\lambda_{\mathrm{ex}}=405 \mathrm{~nm}\right)$

Figure 2 Fluorescence images of NI1 $\left(2 \mu \mathrm{mol} / \mathrm{L}, \lambda_{\mathrm{ex}}=405 \mathrm{~nm}\right)$ and NI2 $\left(2 \mu \mathrm{mol} / \mathrm{L}, \lambda_{\mathrm{ex}}=405 \mathrm{~nm}\right)$ incubation with HeLa cells
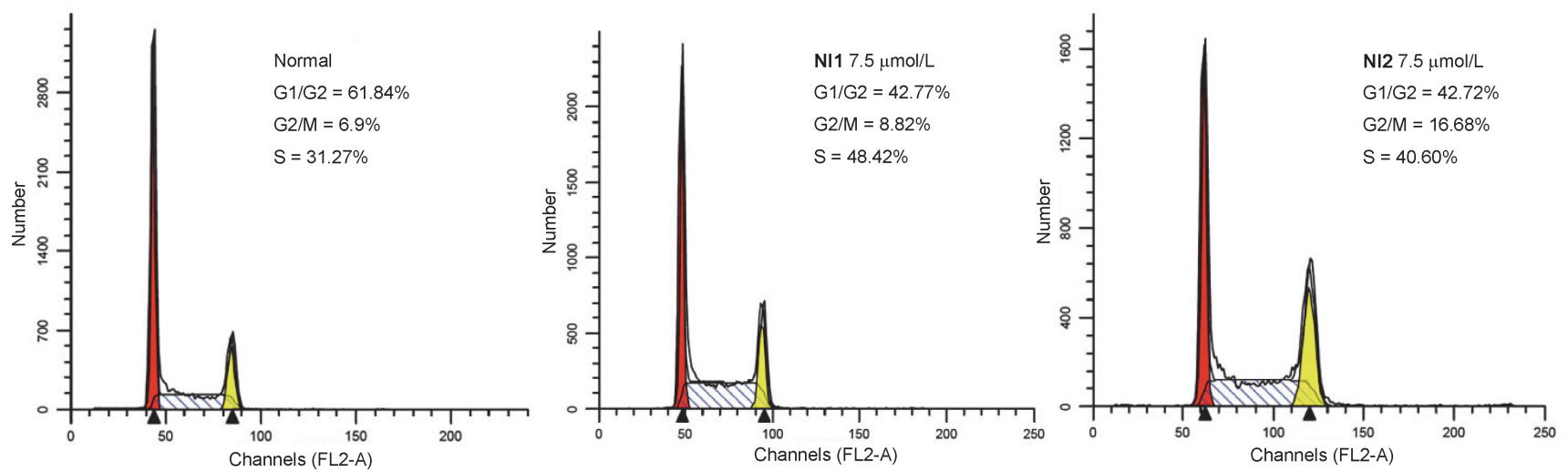

图 $3 \mathrm{HeLa}$ 细胞 $(\mathrm{A})$ 与化合物 NI1 (B) 和 NI2 (C) 作用的细胞周期

Figure 3 Cell cycle analysis of HeLa cells (A) incubation with NI1 (B) and NI2 (C) 
细胞数增加也验证了化合物 NI1 和 NI2 作用于 DNA 分 子，是通过干扰细胞 $\mathrm{S}$ 期发挥其抗肿瘤作用 ${ }^{[25]}$.

\section{6 萗酰亚胺衍生物的血液毒性研究}

根据文献报道, 菜酰亚胺类衍生物虽具有好的抗肿 瘤作用，但其高的血液毒性限制了其临床应用 ${ }^{[26]}$. 因 此，我们研究化合物 NI1 对小鼠的血液毒性.

检测结果如表 2 所示, 各组与正常对照组比较, $P>$ 0.05 , 说明各组数值与正常组均无差异, 即化合物 NI1 对小鼠无急性血液毒性.

\section{2 结论}

合成了系列手性氨基醇修饰的菜酰亚胺类衍生物 NI1 NI8, 化合物结构经 ${ }^{1} \mathrm{H}$ NMR、 ${ }^{13} \mathrm{C}$ NMR 和 HRMS 等表征. 化合物 NI1 NI8 对 HeLa 细胞和 A549 细胞显 示了好的细胞毒性. DNA 络合研究、苂光成像研究和细 胞周期研究结果显示, 部分菜酰亚胺类衍生物作为 DNA 嵌入剂与 DNA 分子有效键合, 并可定位于肿瘤细 胞的细胞核, 其是将肿瘤细胞阻滞于 $\mathrm{S}$ 期达到抑制肿瘤 生长作用. 小鼠血液毒性研究结果说明化合物 NI1 没有 急性血液毒性. 该研究为细胞核定位介导的无血液毒性 的萘酰亚胺类抗肿瘤药物研究做了有益的尝试和探索.

\section{3 实验部分}

\section{1 仪器与试剂}

${ }^{1} \mathrm{H}$ NMR 和 ${ }^{13} \mathrm{C}$ NMR 用 Bruker AVANCE $600 \mathrm{MHz}$ (瑞士 Bruker 公司, 型号 Bruker AV II-600 MHz, TMS 为 内标)测量; 高分辨率质谱用 FTICR-MD (Thermo 公司, 型号 Ionspec 7.0)和 Apex Ultra 测定; 激光共聚焦显微 镜: LSM 800, 德国蔡司(ZEISS); 流式细胞仪: FACSCanto II, 美国 BD 公司; 全自动血细胞分析仪: 美国贝 克曼库尔特 COULTER LH 750/LH 755. 胎牛血清(杭州 四季青生物工程材料有限公司); 改良 Eagle 培养基
$(\mathrm{DMEM})($ 北京索莱宝科技有限公司).

\section{2 化合物的制备}

\subsection{1 中间体 M-1 的合成}

在 $100 \mathrm{~mL}$ 单口瓶中加入 $5 \mathrm{~g}$ (36 mmol) 4-溴-1,8-菜 二甲酸酐, $2 \mathrm{~mL}(36 \mathrm{mmol}) N, N$-二甲基乙二胺，用 $50 \mathrm{~mL}$ 乙醇溶解, 在 $\mathrm{N}_{2}$ 保护下回流反应 $12 \mathrm{~h}$, 经薄层色谱 (TLC)监测, 停止反应, 减压除去乙醇, 粗产品经柱色谱 分离, 用 $[V$ (二氯甲烷 $): V($ 甲醇 $)=20: 1]$ 为洗脱剂, 得 到白色固体 $3.9 \mathrm{~g}$, 产率 $62.5 \%{ }^{[21]}$. m.p. $146.2 \sim 149.8{ }^{\circ} \mathrm{C}$ (m.p. $\left.{ }^{[21]} 152.3 \sim 153.5{ }^{\circ} \mathrm{C}\right) ;{ }^{1} \mathrm{H}$ NMR (600 MHz, $\left.\mathrm{CD}_{3} \mathrm{OD}\right)$ $\delta: 8.53(\mathrm{~d}, J=8.1 \mathrm{~Hz}, 1 \mathrm{H}, \operatorname{ArH}), 8.44(\mathrm{~d}, J=7.2 \mathrm{~Hz}, 1 \mathrm{H}$, ArH), 8.27 (d, $J=8.4 \mathrm{~Hz}, 1 \mathrm{H}, \operatorname{ArH}), 7.54$ (t, $J=7.7 \mathrm{~Hz}$, $1 \mathrm{H}, \mathrm{ArH}), 6.80$ (d, $J=8.5 \mathrm{~Hz}, 1 \mathrm{H}, \mathrm{ArH}), 4.41$ (t, $J=6.3$ $\mathrm{Hz}, 2 \mathrm{H}, \mathrm{CH}_{2}$ ), 3.17 (t, J=6.3 Hz, 2H, $\left.\mathrm{CH}_{2}\right), 2.76$ (s, 6H, $\left.\mathrm{CH}_{3}\right) ;{ }^{13} \mathrm{C} \mathrm{NMR}\left(151 \mathrm{MHz}, \mathrm{CDCl}_{3}\right) \delta: 163.56,133.16$, $132.00,131.18,131.04,130.57,130.17,129.01,128.01$, $123.10,122.23,77.23,77.02,76.80,56.96,45.75,38.31$; MS (ESI) $m / z: 347.03[\mathrm{M}+\mathrm{H}]^{+}$.

\subsection{2 化合物 NI1 NI8 的合成}

在 $50 \mathrm{~mL}$ 圆底烧瓶中加入 $0.5 \mathrm{~g}$ (1.44 mmol) M-1, 加入 $10 \mathrm{~mL}$ 乙二醇单甲醚溶解, 再加入 $14.4 \mathrm{mmol}$ 的氨 基醇类物质 $[(R / S)$-2-氨基丙醇、 $(R / S)$-1-氨基-2-丙醇、 $(R / S)$-2-氨基丁醇)和 $(R / S)$-3-氨基-1,2-丙二醇]以及 $3 \mathrm{~mL}$ $N, N$-二异丙基乙胺(DIPEA), 反应体系在 $\mathrm{N}_{2}$ 保护下回流 反应 $24 \mathrm{~h}$, 经薄层色谱(TLC)监测反应完全, 停止反应, 冷至室温, 减压除去溶剂, 粗产品经柱色谱 $[V($ 二氯甲 烷) $: V($ 甲醇 $)=8 ： 1$ 分离得到产物. NI1 N N8 的产率分 别是 $51.2 \% 、 48.7 \% 、 54.3 \% 、 53.4 \% 、 49.7 \% 、 48.8 \%$ 、 $50.1 \%$ 和 $52.7 \%$.

( $R$ )-2-(2-(二甲基氨基)乙基)-6-((1-差基-2-丙烷基)氨 基)-菜酰亚胺(NI1): 浅黄色固体, m.p. 145.4 148.8 ${ }^{\circ} \mathrm{C}$; $[\alpha]_{\mathrm{D}}^{25}-10\left(c 1.0, \mathrm{CH}_{3} \mathrm{OH}\right) ;{ }^{1} \mathrm{H} \mathrm{NMR}\left(\mathrm{CDCl}_{3}, 600 \mathrm{MHz}\right) \delta$ :

表 2 化合物 NI1 对小鼠的血液毒性 $(\bar{x} \pm s)$

Table 2 Hematological toxicity of compound NI1 in mice $(\bar{x} \pm s)$

\begin{tabular}{|c|c|c|c|c|}
\hline 分组 & 浓度/(mg•mL $\left.{ }^{-1}\right)$ & $\mathrm{WBC} /\left(10^{9} \mathrm{~L}^{-1}\right)$ & $\mathrm{RBC} /\left(10^{12} \mathrm{~L}^{-1}\right)$ & $\mathrm{PLT} /\left(10^{9} \mathrm{~L}^{-1}\right)$ \\
\hline Control & 0 & $4.85 \pm 0.11$ & $9.35 \pm 0.66$ & $358.64 \pm 257.71$ \\
\hline \multirow{3}{*}{$24 \mathrm{~h}$} & 5 & $5.94 \pm 0.52^{a}$ & $8.67 \pm 0.29^{a}$ & $550.33 \pm 74.09^{a}$ \\
\hline & 10 & $5.68 \pm 0.26^{a}$ & $9.23 \pm 0.33^{a}$ & $465.33 \pm 34.12^{a}$ \\
\hline & 20 & $5.83 \pm 0.25^{a}$ & $9.43 \pm 0.38^{a}$ & $475.00 \pm 119.80^{a}$ \\
\hline \multirow{3}{*}{$48 \mathrm{~h}$} & 5 & $4.52 \pm 1.19^{a}$ & $9.12 \pm 0.40^{a}$ & $562.33 \pm 9.29^{a}$ \\
\hline & 10 & $5.23 \pm 1.24^{a}$ & $9.44 \pm 1.46^{a}$ & $563.33 \pm 83.34^{a}$ \\
\hline & 20 & $4.57 \pm 0.50^{a}$ & $7.77 \pm 0.87^{a}$ & $720.00 \pm 336.79^{a}$ \\
\hline \multirow{3}{*}{$96 \mathrm{~h}$} & 5 & $5.71 \pm 0.43^{a}$ & $9.16 \pm 0.23^{a}$ & $233.33 \pm 65.61^{a}$ \\
\hline & 10 & $5.63 \pm 0.30^{a}$ & $9.48 \pm 0.12^{a}$ & $320.00 \pm 161.61^{a}$ \\
\hline & 20 & $5.34 \pm 0.51^{a}$ & $6.52 \pm 0.22^{a}$ & $927.00 \pm 213.55^{a}$ \\
\hline
\end{tabular}

\footnotetext{
${ }^{a} P>0.05$ vs control group.
} 
$1.28\left(\mathrm{~d}, J=6.6 \mathrm{~Hz}, 3 \mathrm{H}, \mathrm{CH}_{3}\right), 2.34\left[\mathrm{~s}, 6 \mathrm{H}, \mathrm{N}\left(\mathrm{CH}_{3}\right)_{2}\right]$, $2.34 \sim 2.66\left(\mathrm{~m}, 2 \mathrm{H}, \mathrm{CH}_{2}\right), 3.69(\mathrm{dd}, J=4.8,10.8 \mathrm{~Hz}, 1 \mathrm{H})$, $3.67 \sim 3.87(\mathrm{~m}, 1 \mathrm{H}), 3.87(\mathrm{dd}, J=3.6,10.8 \mathrm{~Hz}, 1 \mathrm{H})$, $4.13 \sim 4.21\left(\mathrm{~m}, 2 \mathrm{H}, \mathrm{CH}_{2}\right), 5.66(\mathrm{~d}, J=6.6 \mathrm{~Hz}, 1 \mathrm{H}, \mathrm{NH})$, $6.57(\mathrm{~d}, J=8.4 \mathrm{~Hz}, 1 \mathrm{H}, \operatorname{ArH}), 7.39(\mathrm{t}, J=7.8 \mathrm{~Hz}, 1 \mathrm{H}$, ArH), 8.03 (d, $J=8.4 \mathrm{~Hz}, 1 \mathrm{H}, \mathrm{ArH}), 8.21$ (d, $J=8.4 \mathrm{~Hz}$, $1 \mathrm{H}, \operatorname{ArH}), 8.32(\mathrm{~d}, J=7.8 \mathrm{~Hz}, 1 \mathrm{H}, \operatorname{ArH}) ;{ }^{13} \mathrm{C} \mathrm{NMR}$ $\left(\mathrm{CDCl}_{3}, 150 \mathrm{MHz}\right) \delta$ : $16.8,29.7,37.6,45.6,50.1,56.9$, $65.2,104.7,109.7,120.4,122.7,124.5,126.4,129.8$, 131.2, 134.4, 149.1, 164.1, 164.6; HRMS (ESI) calcd for $\mathrm{C}_{19} \mathrm{H}_{24} \mathrm{~N}_{3} \mathrm{O}_{3} 342.1818$, found 342.1816 .

(S)-2-(2-(二甲基氨基)乙基)-6-((1-差基-2-丙烷基)氨 基)-萗酰亚胺(NI2): 浅黄色固体, m.p. 143.2 147.4 ${ }^{\circ} \mathrm{C}$; $[\alpha]_{\mathrm{D}}^{25}+11\left(c 1.0, \mathrm{CH}_{3} \mathrm{OH}\right) ;{ }^{1} \mathrm{H}$ NMR $\left(\mathrm{CDCl}_{3}, 600 \mathrm{MHz}\right) \delta$ : $1.38\left(\mathrm{~d}, J=6.6 \mathrm{~Hz}, 3 \mathrm{H}, \mathrm{CH}_{3}\right), 2.49(\mathrm{~s}, 6 \mathrm{H}), 2.49 \sim 2.82(\mathrm{~m}$, $\left.2 \mathrm{H}, \mathrm{CH}_{2}\right), 3.79(\mathrm{dd}, J=4.8,10.8 \mathrm{~Hz}, 1 \mathrm{H}), 3.93 \sim 3.98(\mathrm{~m}$, $1 \mathrm{H}), 3.97(\mathrm{dd}, J=3.6,11.4 \mathrm{~Hz}, 1 \mathrm{H}), 4.28(\mathrm{t}, J=6.6 \mathrm{~Hz}$, $\left.2 \mathrm{H}, \mathrm{CH}_{2}\right), 5.85(\mathrm{~d}, J=6.0 \mathrm{~Hz}, \mathrm{NH}), 6.66(\mathrm{~d}, J=9.0 \mathrm{~Hz}$, 1H, ArH), 7.46 (t, $J=8.4 \mathrm{~Hz}, 1 \mathrm{H}, \operatorname{ArH}), 8.12$ (d, $J=8.4$ $\mathrm{Hz}, 1 \mathrm{H}, \mathrm{ArH}), 8.26$ (d, $J=8.4 \mathrm{~Hz}, 1 \mathrm{H}, \mathrm{ArH}), 8.37$ (d, $J=$ $7.2 \mathrm{~Hz}, 1 \mathrm{H}, \mathrm{ArH}) ;{ }^{13} \mathrm{C} \mathrm{NMR}\left(\mathrm{CDCl}_{3}, 150 \mathrm{MHz}\right) \delta: 15.8$, 28.7, 36.6, 44.5, 49.2, 55.9, 64.1, 103.7, 108.4, 119.3, $121.5,123.4,125.49,128.7,130.1,133.4,148.2,163.0$, 163.5; HRMS (ESI) calcd for $\mathrm{C}_{19} \mathrm{H}_{24} \mathrm{~N}_{3} \mathrm{O}_{3} 342.1818$, found 342.1816.

(S)-2-(2-(二甲基氨基)乙基)-6-((2-羟丙基)氨基)- $1 H$ 苯并异喹啉-1,3(2H)-二酮 (NI3): 浅黄色固体, m.p. $142.4 \sim 146.6{ }^{\circ} \mathrm{C} ;[\alpha]_{\mathrm{D}}^{25}+22\left(c 1.0, \mathrm{CH}_{3} \mathrm{OH}\right) ;{ }^{1} \mathrm{H} \mathrm{NMR}$ $\left(\mathrm{CDCl}_{3}, 600 \mathrm{MHz}\right) \delta: 1.36\left(\mathrm{~d}, J=6.0 \mathrm{~Hz}, 3 \mathrm{H}, \mathrm{CH}_{3}\right), 2.42$ [s, $\left.6 \mathrm{H}, \mathrm{N}\left(\mathrm{CH}_{3}\right)_{2}\right], 2.72\left(\mathrm{t}, J=6.6 \mathrm{~Hz}, 2 \mathrm{H}, \mathrm{CH}_{2}\right), 3.06 \sim 3.10$ $(\mathrm{m}, 1 \mathrm{H}), 3.29 \sim 3.32(\mathrm{~m}, 1 \mathrm{H}), 4.18 \sim 4.27(\mathrm{~m}, 1 \mathrm{H}), 4.26(\mathrm{t}$, $\left.J=7.2 \mathrm{~Hz}, 2 \mathrm{H}, \mathrm{CH}_{2}\right), 5.97$ (d, $\left.J=3.6 \mathrm{~Hz}, \mathrm{CONH}\right), 6.44$ (d, $J=8.4 \mathrm{~Hz}, 1 \mathrm{H}, \mathrm{ArH}), 7.36(\mathrm{t}, J=8.4 \mathrm{~Hz}, 1 \mathrm{H}, \mathrm{ArH}), 7.97$ (d, $J=8.4 \mathrm{~Hz}, 1 \mathrm{H}, \mathrm{ArH}), 8.22(\mathrm{~d}, J=8.4 \mathrm{~Hz}, 1 \mathrm{H}, \mathrm{ArH})$, $8.31(\mathrm{~d}, J=7.2 \mathrm{~Hz}, 1 \mathrm{H}, \mathrm{ArH}) ;{ }^{13} \mathrm{C}$ NMR $\left(\mathrm{CDCl}_{3}, 150\right.$ MHz) $\delta: 21.6,37.7,45.6,50.6,57.08,65.7,104.4,110.2$, $120.3,122.8,124.6,129.7,131.1,134.6,149.7,164.2$, 164.7; HRMS (ESI) calcd for $\mathrm{C}_{19} \mathrm{H}_{24} \mathrm{~N}_{3} \mathrm{O}_{3} 342.1818$, found 342.1815 .

$(R)-2$-(2-(二甲基氨基) 乙基)-6-((2-羟丙基)氨 基)-1 $H$-苯异喹啉-1,3(2H)-二酮(NI4): 浅黄色固体, m.p. $145.4 \sim 148.8{ }^{\circ} \mathrm{C} ;[\alpha]_{\mathrm{D}}^{25}-24\left(c 1.0, \mathrm{CH}_{3} \mathrm{OH}\right) ;{ }^{1} \mathrm{H} \mathrm{NMR}$ $\left(\mathrm{CDCl}_{3}, 600 \mathrm{MHz}\right) \delta: 1.37\left(\mathrm{~d}, J=6.0 \mathrm{~Hz}, 3 \mathrm{H}, \mathrm{CH}_{3}\right), 2.40$ [s, $6 \mathrm{H}, \mathrm{N}\left(\mathrm{CH}_{3}\right)_{2}$ ], $2.71\left(\mathrm{t}, J=6.6 \mathrm{~Hz}, 2 \mathrm{H}, \mathrm{CH}_{2}\right), 3.12 \sim 3.16$ (m, $1 \mathrm{H}), 3.36 \sim 3.40(\mathrm{~m}, 1 \mathrm{H}), 4.19 \sim 4.30(\mathrm{~m}, 1 \mathrm{H}), 4.29(\mathrm{t}$,
$\left.J=6.6 \mathrm{~Hz}, 2 \mathrm{H}, \mathrm{CH}_{2}\right), 5.81(\mathrm{~s}, 1 \mathrm{H}, \mathrm{CONH}), 6.56$ (d, $J=8.4$ $\mathrm{Hz}, 1 \mathrm{H}, \mathrm{ArH}), 7.49$ (t, $J=7.8 \mathrm{~Hz}, 1 \mathrm{H}, \mathrm{ArH}), 8.03$ (d, $J=$ $8.4 \mathrm{~Hz}, 1 \mathrm{H}, \mathrm{ArH}), 8.34$ (d, $J=8.4 \mathrm{~Hz}, 1 \mathrm{H}, \operatorname{ArH}), 8.45$ (d, $J=7.2 \mathrm{~Hz}, 1 \mathrm{H}, \mathrm{ArH}) ;{ }^{13} \mathrm{C} \mathrm{NMR}\left(\mathrm{CDCl}_{3}, 150 \mathrm{MHz}\right) \delta$ : 21.5, 37.6, 45.6, 50.7, 57.08, 65.3, 104.3, 109.6, 120.2, $122.4,124.4,126.5,129.5,130.9,134.3,149.9,164.2$, 164.6; HRMS (ESI) calcd for $\mathrm{C}_{19} \mathrm{H}_{24} \mathrm{~N}_{3} \mathrm{O}_{3} 342.1818$, found 342.1816 .

(R)-2-(2-(二甲基氨基)乙基)-6-((1-差基-2-丙烷基)氨 基)- $1 H$ - 苯并异喹啉-1,3(2H)-二酮(NI5): 浅黄色固体, m.p. 144.6 $149.6{ }^{\circ} \mathrm{C} ;[\alpha]_{\mathrm{D}}^{25}+21$ (c 1.0, DMSO); ${ }^{1} \mathrm{H}$ NMR (DMSO- $\left.d_{6}+\mathrm{D}_{2} \mathrm{O}, 400 \mathrm{MHz}\right) \delta: 0.95(\mathrm{t}, J=7.2 \mathrm{~Hz}$, $3 \mathrm{H}), 1.64 \sim 1.72(\mathrm{~m}, 2 \mathrm{H}), 2.22(\mathrm{~s}, 6 \mathrm{H}), 2.49$ (d, $J=6.8 \mathrm{~Hz}$ $2 \mathrm{H}), 3.57$ (dd, $J=5.6,6.0 \mathrm{~Hz}, 2 \mathrm{H}), 4.13(\mathrm{t}, J=7.2 \mathrm{~Hz}$, 2H), 6.87 (d, $J=8.8 \mathrm{~Hz}, 1 \mathrm{H}, \mathrm{ArH}), 7.68(\mathrm{t}, J=7.2 \mathrm{~Hz}, 1 \mathrm{H}$, ArH), $8.24(\mathrm{~d}, J=8.4 \mathrm{~Hz}, 1 \mathrm{H}, \mathrm{ArH}), 8.44(\mathrm{~d}, J=7.2 \mathrm{~Hz}$, $1 \mathrm{H}, \operatorname{ArH}), 8.82(\mathrm{~d}, J=8.4 \mathrm{~Hz}, 1 \mathrm{H}, \mathrm{ArH}) ;{ }^{13} \mathrm{C} \mathrm{NMR}$ (DMSO- $d_{6}+\mathrm{D}_{2} \mathrm{O}, 100 \mathrm{MHz}$ ) $\delta: 11.1,24.0,37.4,39.8$, 45.6, 57.0, 62.9, 104.7, 107.6, 120.5, 122.2, 124.6, 129.4, $130.1,131.3,134.8,151.5,163.4,164.3$; HRMS (ESI): calcd. for $\mathrm{C}_{20} \mathrm{H}_{26} \mathrm{~N}_{3} \mathrm{O}_{3} 356.1974$, found 356.1967 .

(S)-2-(2-(二甲基氨基)乙基)-6-((1-羟基-2-丙烷基)氨 基)- $1 H$ - 苯并异喹啉-1,3(2H)-二酮(NI6): 浅黄色固体, m.p. $145.2 \sim 150.8{ }^{\circ} \mathrm{C} ;[\alpha]_{\mathrm{D}}^{25}-20$ (c 1.0, DMSO); ${ }^{1} \mathrm{H}$ NMR (DMSO- $\left.d_{6}+\mathrm{D}_{2} \mathrm{O}, 400 \mathrm{MHz}\right) \delta: 0.94(\mathrm{t}, J=7.2 \mathrm{~Hz}$, $3 \mathrm{H}), 1.25 \sim 1.82(\mathrm{~m}, 2 \mathrm{H}), 2.20$ (s, $6 \mathrm{H}), 2.47$ (t, $J=6.8 \mathrm{~Hz}$, $2 \mathrm{H}), 3.58(\mathrm{~d}, J=5.2 \mathrm{~Hz}, 2 \mathrm{H}), 4.12(\mathrm{t}, J=6.8 \mathrm{~Hz}, 2 \mathrm{H}), 6.87$ (d, $J=8.8 \mathrm{~Hz}, 1 \mathrm{H}, \mathrm{ArH}), 7.68$ (t, $J=7.6 \mathrm{~Hz}, 1 \mathrm{H}, \mathrm{ArH})$, $8.24(\mathrm{~d}, J=8.8 \mathrm{~Hz}, 1 \mathrm{H}, \mathrm{ArH}), 8.44(\mathrm{~d}, J=7.2 \mathrm{~Hz}, 1 \mathrm{H}$, ArH), 8.80 (d, $J=8.4 \mathrm{~Hz}, 1 \mathrm{H}, \mathrm{ArH}) ;{ }^{13} \mathrm{C}$ NMR (DMSO- $d_{6}$ $\left.+\mathrm{D}_{2} \mathrm{O}, 100 \mathrm{MHz}\right) \delta$ : 11.1, 24.0, 37.6, 39.7, 45.8, 56.8, $62.9,104.7,107.6,120.5,122.2,124.6,129.3,130.0$, 131.3, 134.8, 151.4, 163.4, 164.3; HRMS (ESI) calcd for $\mathrm{C}_{20} \mathrm{H}_{26} \mathrm{~N}_{3} \mathrm{O}_{3} 356.1974$, found 356.1967 .

(S)-6-((2,3-二羟丙基)氨基)-2-(2-(二甲基氨基)乙 基)-1 $H$-苯并异喹啉-1,3(2H)-二酮(NI7): 浅黄色固体, m.p. $146.2 \sim 149.4{ }^{\circ} \mathrm{C} ;[\alpha]_{\mathrm{D}}^{25}-23\left(c \quad 1.0, \mathrm{CH}_{3} \mathrm{OH}\right) ;{ }^{1} \mathrm{H}$ NMR (DMSO- $\left.d_{6}+\mathrm{D}_{2} \mathrm{O}, 400 \mathrm{MHz}\right) \delta: 2.38[\mathrm{~s}, 6 \mathrm{H}$, $\left.\mathrm{N}\left(\mathrm{CH}_{3}\right)_{2}\right], 2.52\left(\mathrm{t}, J=4.0 \mathrm{~Hz}, 2 \mathrm{H}, \mathrm{CH}_{2}\right), 2.71(\mathrm{t}, J=8.0 \mathrm{~Hz}$, $\left.2 \mathrm{H}, \mathrm{CH}_{2}\right), 3.47\left(\mathrm{t}, J=4 \mathrm{~Hz}, 2 \mathrm{H}, \mathrm{CH}_{2}\right), 3.85(\mathrm{t}, J=4.0 \mathrm{~Hz}$, $1 \mathrm{H}, \mathrm{NH}), 4.17$ (t, $\left.J=8.0 \mathrm{~Hz}, 2 \mathrm{H}, \mathrm{CH}_{2}\right), 6.82(\mathrm{~d}, J=8.0 \mathrm{~Hz}$, $1 \mathrm{H}, \operatorname{ArH}), 7.67$ (t, $J=8.0 \mathrm{~Hz}, 1 \mathrm{H}, \operatorname{ArH}), 8.24$ (d, $J=8.0$ $\mathrm{Hz}, 1 \mathrm{H}, \mathrm{ArH}), 8.42(\mathrm{~d}, J=8.0 \mathrm{~Hz}, 1 \mathrm{H}, \mathrm{ArH}), 8.72(\mathrm{~d}, J=$ $8.0 \mathrm{~Hz}, 1 \mathrm{H}, \mathrm{ArH}$ ); ${ }^{13} \mathrm{C}$ NMR (DMSO- $d_{6}+\mathrm{D}_{2} \mathrm{O}, 100 \mathrm{MHz}$ ) $\delta: 37.2,45.3,46.8,56.8,64.3,69.7,104.5,107.8,120.5$, 
$122.2,124.8,129.2,129.9,131.3,134.8,151.5,163.6$, 164.5; HRMS (ESI) calcd for $\mathrm{C}_{19} \mathrm{H}_{24} \mathrm{~N}_{3} \mathrm{O}_{4} 358.1767$, found 358.1760 .

(R)-6-((2,3-二羟丙基)氨基)-2-(2-(二甲基氨基)乙 基)-1 $H$-苯并异喹啉-1,3(2H)-二酮(NI8): 浅黄色固体, m.p. $143.2 \sim 147.6{ }^{\circ} \mathrm{C} ;[\alpha]_{\mathrm{D}}^{25}+21\left(c 1.0, \mathrm{CH}_{3} \mathrm{OH}\right) ;{ }^{1} \mathrm{H}$ NMR (DMSO- $\left.d_{6}+\mathrm{D}_{2} \mathrm{O}, 400 \mathrm{MHz}\right) \delta: 2.38[\mathrm{~s}, 6 \mathrm{H}$, $\left.\mathrm{N}\left(\mathrm{CH}_{3}\right)_{2}\right], 2.52\left(\mathrm{t}, J=4.0 \mathrm{~Hz}, 2 \mathrm{H}, \mathrm{CH}_{2}\right), 2.71(\mathrm{t}, J=8.0 \mathrm{~Hz}$, $\left.2 \mathrm{H}, \mathrm{CH}_{2}\right), 3.47\left(\mathrm{t}, J=4.0 \mathrm{~Hz}, 2 \mathrm{H}, \mathrm{CH}_{2}\right), 3.85(\mathrm{t}, J=4.0 \mathrm{~Hz}$, $1 \mathrm{H}, \mathrm{NH}), 4.17$ (t, $\left.J=8.0 \mathrm{~Hz}, 2 \mathrm{H}, \mathrm{CH}_{2}\right), 6.82(\mathrm{~d}, J=8.0 \mathrm{~Hz}$, $1 \mathrm{H}, \operatorname{ArH}), 7.67$ (t, $J=8.0 \mathrm{~Hz}, 1 \mathrm{H}, \operatorname{ArH}), 8.24$ (d, $J=8.0$ $\mathrm{Hz}, 1 \mathrm{H}, \mathrm{ArH}), 8.42(\mathrm{~d}, J=8.0 \mathrm{~Hz}, 1 \mathrm{H}, \mathrm{ArH}), 8.72(\mathrm{~d}, J=$ $8.0 \mathrm{~Hz}, 1 \mathrm{H}, \mathrm{ArH}$ ); ${ }^{13} \mathrm{C}$ NMR (DMSO- $d_{6}+\mathrm{D}_{2} \mathrm{O}, 100 \mathrm{MHz}$ ) $\delta: 37.0,45.2,46.8,56.7,64.3,69.7,104.5,107.8,120.5$, $122.2,124.8,129.2,129.9,131.3,134.8,151.5,163.6$, 164.4; HRMS (ESI) calcd for $\mathrm{C}_{19} \mathrm{H}_{24} \mathrm{~N}_{3} \mathrm{O}_{4} 358.1767$, found 358.1762 .

\section{3 细胞毒性研究}

将肿瘤细胞(人宫颈癌细胞 HeLa、人乳腺癌细胞 MCF-7、人肺癌细胞 A549 和人胃癌细胞 MGC-803)接 种于 96 孔板 (细胞 $7 \times 10^{3} /$ 孔, 改良 Eagle 培养基 (DMEM) 完全培养液 $180 \mu \mathrm{L} /$ 孔), $37{ }^{\circ} \mathrm{C}$, 体积分数为 $5 \%$ 的 $\mathrm{CO}_{2}$, 贴壁过夜. 次日, 加化合物, 体积为 $20 \mu \mathrm{L} /$ 孔 (终浓度分别为 $0.2 、 1 、 5 、 25$ 和 $125 \mu \mathrm{mol} / \mathrm{L}$ ), 每个浓度 3 个复孔, 加化合物 $44 \mathrm{~h}$ 后加 MTT $(20 \mu \mathrm{L} /$ 孔 $), 48 \mathrm{~h}$ 后弃 上清, 加二甲基亚砜(DMSO) (150 $\mu \mathrm{L} /$ 孔), 经酶标仪检 测 $490 \mathrm{~nm}$ 吸光度值.

\section{4 萗酰亚胺类衍生物与 Ct-DNA 作用研究}

NI1 和 NI2 浓度为 $1 \times 10^{-5} \mathrm{~mol} / \mathrm{L}$, 分别加入 $0 \sim$ $3 \times 10^{-4} \mathrm{~mol} / \mathrm{L}$ 浓度的 Ct-DNA, 磷酸盐缓冲溶液(PBS, $10 \mathrm{mmol} / \mathrm{L}, \mathrm{pH} 7.4,50 \mathrm{mmol} / \mathrm{L} \mathrm{NaCl}$ ) 定容 $(5 \mathrm{~mL})$, 混匀, 静置 $2 \mathrm{~h}$, 在 300 600 nm 范围下检测紫外吸收, 采样间 隔 $0.2 \mathrm{~nm}$, 扫描速度中速.

NI1 和 NI2 浓度为 $1 \times 10^{-5} \mathrm{~mol} / \mathrm{L}$, 加入 $0 \sim 3 \times$ $10^{-4} \mathrm{~mol} / \mathrm{L}$ 浓度的 Ct-DNA, PBS (10 mmol/L, pH 7.4, 50 $\mathrm{mmol} / \mathrm{L} \mathrm{NaCl}$ ) 定容 $(5 \mathrm{~mL})$, 混匀, 静置 $2 \mathrm{~h}$, 在 $400 \sim 750$ $\mathrm{nm}$ 范围下检测苂光发射.

\section{5 萗酰亚胺类衍生物与 HeLa 细胞荧光成像研究}

将 Hela 细胞种于六孔板 $\left(1.8 \times 10^{5}\right.$ 个/孔 $), 37{ }^{\circ} \mathrm{C}$, 体积分数为 $5 \%$ 的 $\mathrm{CO}_{2}$ 培养贴壁过夜. 次日, 分别加浓 度为 $20 \mu \mathrm{mol} / \mathrm{L}$ 的 NI1 和 NI2 $(200 \mu \mathrm{L} /$ 孔 $), 1.8 \mathrm{~mL} /$ 孔 DMEM 培养液, $2 \mathrm{~h}$ 后进行共聚焦成像.

\section{6 萗酰亚胺类衍生物与 HeLa 细胞的周期研究}

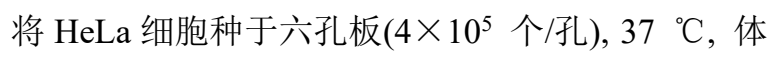

积分数为 $5 \%$ 的 $\mathrm{CO}_{2}$ 培养贴壁过夜. 次日, 分别加不同 浓度的 NI1 和 NI2 (200 $\mu \mathrm{L}$ 孔), $1.8 \mathrm{~mL}$ DMEM 培养液, $12 \mathrm{~h}$ 后收集孔内所有细胞, 离心(1200 r/min, $3 \mathrm{~min}), \mathrm{PBS}$ 缓冲液洗涤 2 遍. 使用 $\mathrm{BD}$ 周期试剂盒进行周期检测. 经流式细胞仪检测细胞周期变化.

\section{7 萗酰亚胺类衍生物的血液毒性研究}

将 $4 \sim 6$ 周的昆明小鼠称重后随机分组, 分为 10 组, 每组 10 只: 正常对照组、剂量分别为 $5 、 10$ 和 $20 \mathrm{mg} / \mathrm{mL}$ 作用 $24 \mathrm{~h}$ 组、 $48 \mathrm{~h}$ 组和 $96 \mathrm{~h}$ 组. 经小鼠腹腔注射方式给 药, 给药体积为 $1 \mathrm{~mL}$, 我们分别于给药后 $24 、 48$ 和 $96 \mathrm{~h}$ 眼球取血, 静置 $30 \mathrm{~min}$, 离心 $(2000 \mathrm{r} / \mathrm{min}, 5 \mathrm{~min})$, 经全 自动血细胞分析仪检测红细胞( $\mathrm{RBC})$ 、白细胞(WBC)和 血小板(PLT)的数量 ${ }^{[27]}$. 数据经 SPSS19.0 软件分析, 统 计学方法采用单因素方差分析和 $\mathrm{t}$ 检验及独立样本 $\mathrm{t}$ 检 验. 检验水准 $\alpha=0.05, P<0.05$ 表示有显著性差异.

Supporting Information 化合物 NI1 NI8 的 ${ }^{1} \mathrm{H}$ NMR、 ${ }^{13} \mathrm{C}$ NMR、HRMS、MS 和苂光光谱等. 这些材 料可以免费从本刊网站(http://sioc-journal.cn/)上下载.

\section{References}

[1] Tiwari, R.; Jain, P.; Asati, S.; Haider, T.; Soni, V.; Pandey, V. J. Drug Delivery Sci. Technol. 2018, 48, 383.

[2] Wang, G. H.; Chen, H.; Cai, Y. Y.; Li, L.; Yang, H. K.; Li, Q.; He, Z. J.; Lin, J. T. Mater. Sci. Eng. C 2018, 90, 568.

[3] (a) Ahmad, M. Z.; Akhter, S.; Rahman, Z.; Akhter, S.; Anwar, M.; Mallik, N.; Ahmad, F. J. J. Pharm. Pharmacol. 2013, 65, 634. (b) Ali, M. R. K.; Wu, Y.; Ghosh, D.; Do, B. H.; Chen, K.; Dawson, M. R.; Fang, N.; Sulchek, T. A.; El-Sayed, M. A. ACS Nano 2017, 11,3716 .

[4] (a) Pan, L. M.; Shi, J. L. Chin. J. Chem. 2018, 36, 481. (b) Marshall, W. F. Curr. Biol. 2002, 12, 185.

[5] Dey, P. Diagn. Cytopathol. 2010, 38, 382.

[6] Zink, D.; Fischer, A. H.; Nickerson, J. A. Nat. Rev. Cancer 2004, 4, 677.

[7] Peng, H. B.; Tang, J.; Zheng, R.; Guo, G. N.; Dong, A. G.; Wang, Y J.; Yang, W. L. Adv. Healthcare Mater. 2017, 6, 1601289.

[8] (a) Michalak, E. M.; Burr, M. L.; Bannister, A. J.; Dawson, M. A. Nat. Rev. Mol. Cell Biol. 2019, 20, 573.

(b) Tandon, R.; Luxami, V.; Kaur, H.; Tandon, K.; Paul, K. Chem. Rec. 2017, 17, 956.

[9] Zhou, J.; Chang, A.; Wang, L. L.; Liu, Y.; Liu, X. J.; Shangguan, D H. Org. Biomol. Chem. 2014, 56, 9207.

[10] Zhang, H.; Wang, K.; Xuan, X. P.; Lv, Q. Z.; Nie, Y. M.; Guo, H. M. Chem. Commun. 2016, 52, 6308.

[11] Singh, K.; Rotaru, A. M.; Beharry, A. A. ACS Chem. Biol. 2018, 13, 1785.

[12] Skok, Ž.; Zidar, N.; Kikelj, D.; Ilaš, J. J. Med. Chem. 2020, 63, 884.

[13] Liu, J.; Liu, X. J.; Lu, S. S.; Zhang, L. L.; Feng, L.; Zhong, S. L.; Zhang, N.; Bing, T.; Shangguan, D. H. Analyst 2020, 145, 6549.

[14] Fueyo-González, F.; Fernández-Gutiérrez, M.; García-Puentes, D.; Orte, A.; González-Vera, J. A.; Herranz, R. Eur. J. Med. Chem., 2020, 200, 112407.

[15] Wu, Z.; Liu, M.; Liu, Z.; Tian, Y. J. Am. Chem. Soc. 2020, 142, 7532.

[16] Feng, H.; Meng, Q. T.; Ta, H. T.; Zhang, R. New J. Chem. 2020, 44, 12890. 
[17] Wang, K. R.; Qian, F.; Sun, Q.; Ma, C. L.; Rong, R. X.; Cao, Z. R.; Wang, X. M.; Li, X. L. Chem. Biol. Drug Des. 2016, 87, 664.

[18] Rong, R. X.; Wang, S. S.; Liu, X.; Li, R. F.; Wang, K. R.; Cao, Z. R.; Li, X. L. Bioorg. Med. Chem. Lett. 2018, 28, 742.

[19] Wang, K. R.; Qian, F.; Rong, R. X.; Cao, Z. R.; Wang, X. M.; Li, X. L. $R S C A d v$. 2014, 4, 47605 .

[20] Rong, R. X.; Sun, Q.; Ma, C. L.; Chen, B.; Wang, W. Y.; Wang, Z. A.; Wang, K. R.; Li, X. L. Med. Chem. Commun. 2016, 7, 679.

[21] Wang, K. R.; An, H. W.; Wang, Y. Q.; Yan, X. H.; Li, Y.; Chen, H.; Zhang, P. Z.; Li, J. M.; Li, X. L.; Zhang, J. C. Chin. J. Org. Chem. 2012, 32, 696 (in Chinese).

(王克让, 安红维, 王月清, 间新豪, 李锐, 陈华, 张平竹, 李金 梅, 李小六, 张金超, 有机化学, 2012, 32, 696.)

[22] Sharma, H.; Sidhu, J. S.; Hassen, W. M.; Singh, N.; Dubowski, J. J. ACS Omega 2019, 4, 5829.

[23] Elmes, R. B. P.; Ryan, G. J.; Erby, M. L.; Frimannsson, D. O.;
Kitchen, J. A.; Lawler, M.; Williams, D. C.; Quinn, S. J.; Gunnlaugsson, T. Inorg. Chem. 2020, 59, 10874.

[24] Petrova, N. V.; Velichko, A. K.; Razin, S. V.; Kantidze, O. L. Cell Cycle 2016, 15, 337

[25] (a) Bai, T.; Zhu, B.; Shao, D.; Lian, Z.; Liu, P.; Shi, J.; Kong, J. Macromol. Biosci. 2020, 20, e1900438.

(b) Wang, S. S.; Zhang, Q. L.; Chu, P.; Kong, L. Q.; Li, G. Z.; Li, Y. Q.; Yang, L.; Zhao, W. J.; Guo, X. H.; Tang, Z. Y. Bioorg. Chem. 2020, 101,104036 .

[26] Wang, K. R.; Qian, F.; Wang, X. M.; Tan, G. H.; Rong, R. X.; Cao, Z. R.; Chen, H.; Zhang, P. Z.; Li, X. L. Chin. Chem. Lett. 2014, 25, 1087.

[27] Banerjee, S.; Veale, E. B.; Phelan, C. M.; Murphy, S. A.; Tocci, G. M.; Gillespie, L. J.; Frimannsson, D. O.; Kelly, J. M.; Gunnlaugsson, T. Chem. Soc. Rev. 2013, 42, 1601. 